OPEN ACCESS

Edited by:

Yu-Hui Liu,

Third Military Medical University,

China

Reviewed by:

Yang Yang,

Third Military Medical University,

China

Shu Yu,

Army Medical University, China

*Correspondence:

Jian-Hong Wang

jhwangneurology@yeah.net

Received: 10 June 2020

Accepted: 17 August 2020

Published: 25 September 2020

Citation:

Wang J, Zheng B, Yang S, Hu M and

Wang J-H (2020) Differential

Circulating Levels of Naturally

Occurring Antibody to $\alpha$-Synuclein in

Parkinson's Disease Dementia,

Alzheimer's Disease, and

Vascular Dementia.

Front. Aging Neurosci. 12:571437.

doi: 10.3389/fnagi.2020.571437

\section{Differential Circulating Levels of Naturally Occurring Antibody to $\alpha$-Synuclein in Parkinson's Disease Dementia, Alzheimer's Disease, and Vascular Dementia}

\author{
Jian Wang ${ }^{1}$, Bo Zheng ${ }^{1}$, Shu Yang ${ }^{2}$, Mei Hu ${ }^{3}$ and Jian-Hong Wang ${ }^{2 *}$ \\ 'Department of Neurology, Yaan People's Hospital, Yaan, China, '2Department of Neurology, Sichuan Provincial People's \\ Hospital, University of Electronic Science and Technology of China, Chengdu, China, ${ }^{3}$ Department of Imaging, Sichuan \\ Provincial People's Hospital, University of Electronic Science and Technology of China, Chengdu, China
}

Background: Aggregation of alpha-synuclein ( $\alpha$-Syn) is considered to be a significant pathological hallmark and a driving force of Parkinson's disease (PD). PD dementia (PDD) occurs in a substantial number of PD patients. Naturally occurring antibody against $\alpha$-Syn (NAb- $\alpha$-Syn) exists ubiquitously in human blood and is reported to be altered in PD. However, it is not clear yet whether PDD had similar changes of circulating NAb- $\alpha$-Syn.

Methods: In this study, we recruited 61 PDD patients, 52 patients with Alzheimer's disease (AD), 51 patients with vascular dementia (VaD), and 50 normal controls (NCs). ELISA was used to examine NAb- $\alpha$-Syn levels in serum.

Results: In comparison with NCs, serum levels of NAb- $\alpha$-Syn were significantly lower in patients with PDD. However, serum levels of NAb- $\alpha$-Syn were comparable among $A D, V a D$, and NC groups. Serum levels of NAb- $\alpha$-Syn were positively correlated with the cognitive function, as reflected by Mini-Mental State Examination (MMSE) and Montreal Cognitive Assessment (MoCA). Serum levels of NAb- $\alpha$-Syn were negatively correlated with the severity of PD [as reflected by the Unified Parkinson Disease Rating Scale (UPDRS)] and the duration of PD and PDD. Serum NAb- $\alpha$-Syn can differentiate PDD patients from $\mathrm{AD}$ and $\mathrm{VaD}$ patients.

Conclusion: These results suggest that circulating NAb- $\alpha$-Syn might be a potential biomarker of PDD.

Keywords: $\alpha$-Synuclein, dementia, naturally occurring antibody, biomarker, circulating

\section{INTRODUCTION}

Neurodegenerative disorders such as Parkinson's disease (PD), Alzheimer's disease (AD), and Creutzfeldt-Jakob disease (CJD) are characterized by the deposition and aggregation of misfolded proteins in the central nervous system (CNS; Skovronsky et al., 2006). The characteristic pathological changes in PD consist of dopaminergic neuronal loss, gliosis, and intraneuronal Lewy body formation in the substantia nigra pars compacta and striatum. Alpha-Synuclein $(\alpha-S y n)$ was found to be the predominant protein in Lewy body inclusions (Tofaris and Spillantini, 2007). 
Although PD is considered to be a motor disorder, non-motor symptoms may occur from early stages, even before the manifestation of motor symptoms. Dementia can occur in a substantial number of PD patients with a prevalence of about 30\% (Hanagasi et al., 2017). PD dementia (PDD) is characterized by a dysexecutive syndrome with early and prominent impairment of attention and visuospatial functions, moderately impaired episodic memory, and relatively preserved core language functions (Hanagasi et al., 2017). Although the symptoms and clinical courses vary in PDD and other types of dementia, there is some overlap of clinical characteristics among different dementias. Currently, blood-based biomarkers are limited to distinguish them. After damage of neurons or axons, soluble $\alpha$-Syn is released into the cerebral spinal fluid and efflux to the circulation in PD patients and in healthy individuals (El-Agnaf et al., 2006). This event may stimulate an induction of autoantibody formation against $\alpha$ Syn [naturally occurring antibody against $\alpha$-Syn (NAb- $\alpha-$ Syn); Papachroni et al., 2007]. The levels of circulating NAb$\alpha$-Syn have been reported to be altered in PD patients (Besong-Agbo et al., 2013). However, studies investigating the levels of NAb- $\alpha$-Syn in PDD patients are limited. In this study, we investigated whether circulating NAb- $\alpha$-Syn was capable of distinguishing $\mathrm{PDD}$ from $\mathrm{AD}$ and vascular dementia $(\mathrm{VaD})$.

\section{MATERIALS AND METHODS}

\section{Participants}

A total number of $61 \mathrm{PDD}, 52 \mathrm{AD}$, and $51 \mathrm{VaD}$ patients were consecutively recruited from the Department of Neurology, Sichuan Provincial People's Hospital. Fifty normal controls (NCs) without cognitive dysfunction were recruited from the health examination center of Sichuan Provincial People's Hospital. PDD was diagnosed according to criteria proposed by the Movement Disorder Society Task Force (Emre et al., 2007). Patients who developed dementia within 1 year after PD onset were excluded. AD diagnoses were based on the clinical criteria for probable $\mathrm{AD}$ as described by the National Institute on Aging-Alzheimer's Association (McKhann et al., 2011). The diagnosis of $\mathrm{VaD}$ was based on the Diagnostic and Statistical Manual of Mental Disorders-fourth Edition (DSM-IV) and also fulfilled the imaging criteria for $\mathrm{VaD}$ (Erkinjuntti et al., 2000). Patients with cognitive and behavioral symptoms presenting as a result of other conditions, such as acute confusion due to systemic disease, abnormality or drug intoxication, depression, normal pressure hydrocephalus, progressive supranuclear palsy, or history of significant brain trauma followed by persistent neurologic deficit or known structural brain abnormality, were excluded. All participants received cognitive assessment using Mini-Mental State Examination (MMSE) and Montreal Cognitive Assessment (MoCA). Severity of PD was assessed by the Unified Parkinson Disease Rating Scale (UPDRS). Informed consent was obtained from all patients and their legal relatives. This research project was approved by the institutional review boards at Sichuan Provincial People's Hospital.

\section{Isolation of Naturally Occurring Antibody Against Alpha-Synuclein}

NAb- $\alpha$-Syn isolated from human intravenous immunoglobulin G (IVIg; Yuanda, Sichuan, China) was used as a standard. NAb$\alpha$-Syn was extracted using affinity chromatography. Briefly, a column was packed with $2 \mathrm{ml}$ resin (PIERCE Biotechnology, Rockford, IL, USA), labeled with $1 \mathrm{mg}$ recombinant $\alpha$-Syn (rPeptide, Bogart, GA, USA), equilibrated, and washed with phosphate-buffered saline (PBS, pH 7.4). After passing purified IVIg through the column, fractions were eluted with glycine buffer, $\mathrm{pH}$ 2.8. The main fractions containing the highest amount of NAb- $\alpha$-Syn were pooled, and their concentration was determined using the NanoDrop spectrometer (Nanodrop1000, PeqLab, Erlangen, Germany). Pooled NAb- $\alpha$-Syn was stored at $-20^{\circ} \mathrm{C}$ until use.

\section{Naturally Occurring Antibody Against Alpha-Synuclein ELISA}

High-bind 96-well ELISA plates (Nunc, Denmark) were coated overnight with recombinant $\alpha$-Syn $50 \mu \mathrm{g} / \mathrm{ml}(100 \mathrm{ml} /$ well $)$ in phosphate-coating buffer (1.7 $\mathrm{mM}$ sodium dihydrogen phosphate, $98 \mathrm{mM}$ disodium hydrogen phosphate, $0.05 \%$ sodium azide; $\mathrm{pH} 7.6$ ) at $4^{\circ} \mathrm{C}$. Wells were then blocked with $5 \%$ bovine serum albumin (BSA; Sigma, St. Louis, MO, USA) in PBS for $1 \mathrm{~h}$ at $37^{\circ} \mathrm{C}$. Standards were prepared by diluting purified NAb- $\alpha$-Syn from IVIg in dilution buffer (5\% BSA). Sera were diluted 1:100. Plates were washed four times with $300 \mathrm{ml}$ washing buffer (PBS with $0.05 \%$ Tween-20) and incubated with standards and samples $(100 \mathrm{ml} /$ well $)$ for $1 \mathrm{~h}$ at room temperature (RT). After washing, plates were incubated with $100 \mathrm{ml} /$ well of detection antibody, a 1:5,000 dilution of peroxidase-labeled goat antihuman IgG antibody (Sigma, St. Louis, MO, USA) in dilution buffer, at RT for $1 \mathrm{~h}$. After a final wash, the assay was developed using $100 \mathrm{ml} /$ well TMB (Sigma, St. Louis, MO, USA) for $15 \mathrm{~min}$. The reaction was stopped with $30 \mathrm{ml} 2 \mathrm{~N}$ sulfuric acid (Roth, Karlsruhe, Germany) and read at $450 \mathrm{~nm}$. The difference in signal between coated and uncoated wells was considered to be solely due to $\alpha$-Syn-nAbs binding to $\alpha$-Syn and was used for further calculations.

\section{Statistics}

Statistical analysis was performed using SPSS (version 19.0, SPSS, Chicago, IL, USA). The Kolmogorov-Smirnov and Shapiro-Wilk tests were used to check for normal distribution. To compare demographic, clinical, and serum data between groups, parametric and nonparametric tests such as one-way analysis of variance were used. Kruskal-Wallis test was used to compare multiple groups ( $\mathrm{PDD}, \mathrm{AD}, \mathrm{VaD}$, and $\mathrm{NC}$ ), followed by post hoc pairwise comparisons using Dunn multiple comparison procedures. In order to test for significant correlations between clinical and demographic characteristics of patients and the serum data, Spearman rank correlation was used. Optimal sensitivity and specificity were determined via the receiver operating characteristic (ROC) curve analysis to determine the capacity of NAb- $\alpha$-Syn in differentiating different types of dementia. A $p<0.05$ was regarded as significant. 
TABLE 1 | Demographic data of subjects.

\begin{tabular}{|c|c|c|c|c|c|}
\hline & NC $(n=50)$ & PDD $(n=61)$ & $\mathrm{AD}(n=52)$ & $\operatorname{VaD}(n=51)$ & $p$ \\
\hline Age, years & $66.68 \pm 8.63$ & $69.07 \pm 8.25$ & $66.90 \pm 9.58$ & $65.00 \pm 8.99$ & 0.115 \\
\hline Female, n (\%) & $25(50.00)$ & $31(50.82)$ & 25 (48.08) & $24(47.06)$ & 0.978 \\
\hline ApoE $\varepsilon 4, n(\%)$ & $10(20.00)$ & $12(19.67)$ & $24(46.15)$ & $10(19.61)$ & 0.003 \\
\hline Education, years & $8.84 \pm 4.49$ & $8.92 \pm 4.55$ & $8.08 \pm 4.27$ & $8.47 \pm 4.58$ & 0.752 \\
\hline MMSE & $27.96 \pm 1.88$ & $14.52 \pm 6.62$ & $14.87 \pm 6.21$ & $15.27 \pm 5.37$ & $<0.001$ \\
\hline MoCA & $28.12 \pm 1.76$ & $12.90 \pm 6.24$ & $13.19 \pm 5.15$ & $12.10 \pm 4.61$ & $<0.001$ \\
\hline UPDRS & NA & $29.70 \pm 1.75$ & NA & NA & NA \\
\hline Duration of PD & NA & $7.26 \pm 3.83$ & NA & NA & NA \\
\hline Duration of dementia & NA & $2.57 \pm 2.51$ & $5.73 \pm 2.96$ & $6.04 \pm 3.38$ & $<0.001$ \\
\hline
\end{tabular}

Age, education, MMSE, MoCA, and UPDRS were expressed as means \pm SD. Female and ApoE $\varepsilon 4$ carriers were expressed as number (frequencies). NA, not applicable; NC, normal control; PDD, Parkinson's disease dementia; AD, Alzheimer's disease; VaD, vascular dementia; MMSE, Mini-Mental State Examination; MoCA, Montreal Cognitive Assessment; UPDRS, Unified Parkinson Disease Rating Scale.

\section{RESULTS}

\section{Demographics}

The present study recruited 61 PDD patients, $52 \mathrm{AD}$ patients, $51 \mathrm{VaD}$ patients, and $50 \mathrm{NCs}$. The average age was not significantly different among groups (NC: $66.68 \pm 8.63$ years, PDD: $69.07 \pm 8.25$ years, AD: $66.90 \pm 9.58$ years, $\mathrm{VaD}$ : $65.00 \pm 8.99$ years; $p=0.115)$. The percentages of females were similar among groups (NC: $50.00 \%$, PDD: $50.82 \%$, AD: $48.08 \%, \mathrm{VaD}: 47.06 \%$; $p=0.978$ ). However, the percentage of ApoE $\varepsilon 4$ carriers was significantly higher in the $\mathrm{AD}$ group (NC: 20.00\%, PDD: $19.67 \%$, AD: 46.15\%, VaD: $19.61 \%$; $p=0.003$ ). The average education level was also not significantly different among groups (NC: $8.84 \pm 4.49$ years, PDD: $8.92 \pm 4.55$ years, $\mathrm{AD}: 8.08 \pm 4.27$ years, $\mathrm{VaD}: 8.47 \pm 4.58$ years; $p=0.752$ ). The cognitive status, as reflected by MMSE (NC: $27.96 \pm 1.88$, PDD: $14.52 \pm 6.62, \mathrm{AD}: 14.87 \pm 6.21, \mathrm{VaD}: 15.27 \pm 5.37$; $p<0.001$ ) and MoCA (NC: $28.12 \pm 1.76$, PDD: $12.90 \pm 6.24$, AD: $13.19 \pm 5.15$, VaD: $12.10 \pm 4.61 ; p<0.001$ ), was significantly different among groups (Table 1).

\section{Serum Naturally Occurring Antibody Against Alpha-Synuclein Levels in Different Types of Dementia}

We first investigated serum levels of NAb- $\alpha$-Syn in different types of dementia. We found that serum levels NAb- $\alpha$-Syn were significantly higher in the PDD group $(86.2 \pm 53.6 \mathrm{pg} / \mathrm{ml})$ in comparison with those in the NC $(156.3 \pm 79.4 \mathrm{pg} / \mathrm{ml}$; $p<0.001), \mathrm{AD}(136.9 \pm 48.2 \mathrm{pg} / \mathrm{ml} ; p<0.001)$, and $\mathrm{VaD}$ $(139.7 \pm 56.6 \mathrm{pg} / \mathrm{ml} ; p<0.001)$ groups. However, no significant difference was found among the $\mathrm{NC}, \mathrm{AD}$, and $\mathrm{VaD}$ groups (Figure 1). These findings indicated that the alteration of serum NAb- $\alpha$-Syn levels was relatively specific in PDD.

\section{Correlations of Serum Naturally Occurring Antibody Against Alpha-Synuclein Levels With Cognitive Function}

We next investigated the correlations of serum NAb- $\alpha$-Syn levels with the cognitive functions. We found that serum NAb- $\alpha$-Syn levels were significantly correlated with MMSE in the overall group $(\gamma=0.288, p<0.001)$. Subgroup analysis indicated that serum NAb- $\alpha$-Syn levels were significantly

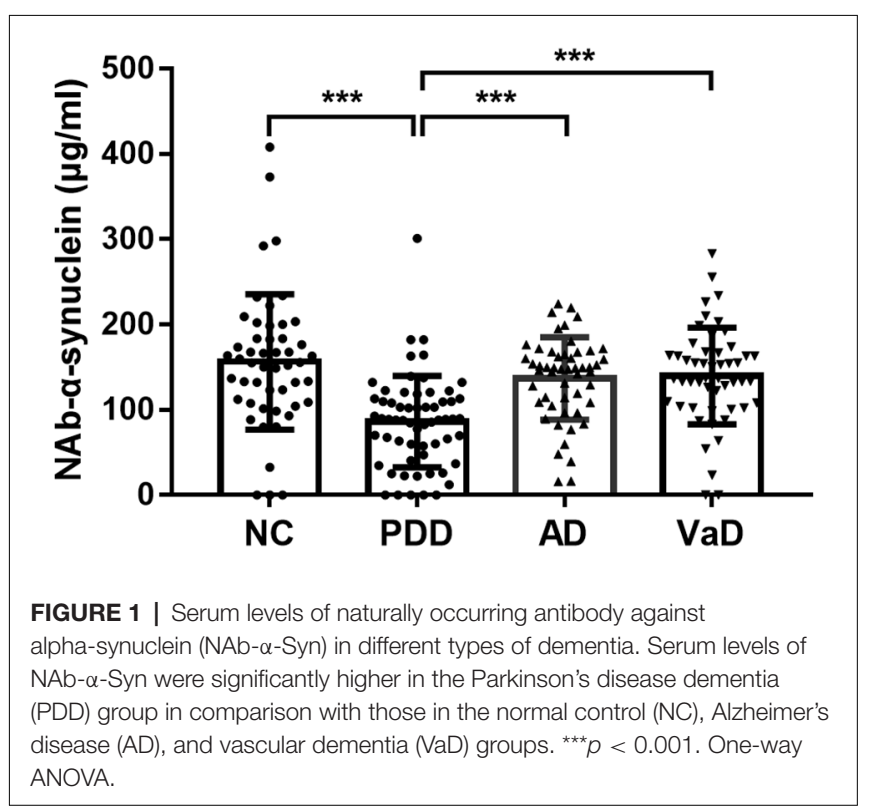

correlated with MMSE in the PDD group $(\gamma=0.342$, $p=0.007)$, but not in the NC $(\gamma=0.079, p=0.585), \mathrm{AD}$ $(\gamma=0.086, p=0.544)$, or $\operatorname{VaD}(\gamma=0.138, p=0.334)$ group (Figure 2). Similarly, serum NAb- $\alpha$-Syn levels were significantly correlated with $\mathrm{MoCA}$ in the overall group $(\gamma=0.260, p<0.001)$ and the PDD subgroup $(\gamma=0.300$, $p=0.019)$, but not in the NC $(\gamma=-0.027, p=0.852)$, $\mathrm{AD}(\gamma=0.013, p=0.929)$, or $\mathrm{VaD}$ group $(\gamma=0.157$, $p=0.273$; Figure 3 ).

\section{Correlations of Serum Naturally Occurring Antibody Against Alpha-Synuclein Levels With Disease Severity and Duration}

We next investigated the correlations between serum NAb$\alpha$-Syn levels and UPDRS in the PDD group. We found that serum NAb- $\alpha$-Syn levels were negatively correlated with UPDRS $(\gamma=-0.531, p<0.001$; Figure 4A). However, serum NAb- $\alpha$ Syn levels were not significantly correlated with the age of PDD patients $(\gamma=-0.096, p=0.463$; Figure 4B). Serum NAb- $\alpha$-Syn levels had a positive correlation with both the duration of $\mathrm{PD}$ $(\gamma=-0.374, p=0.003)$ and dementia $(\gamma=-0.498, p<0.001)$ 

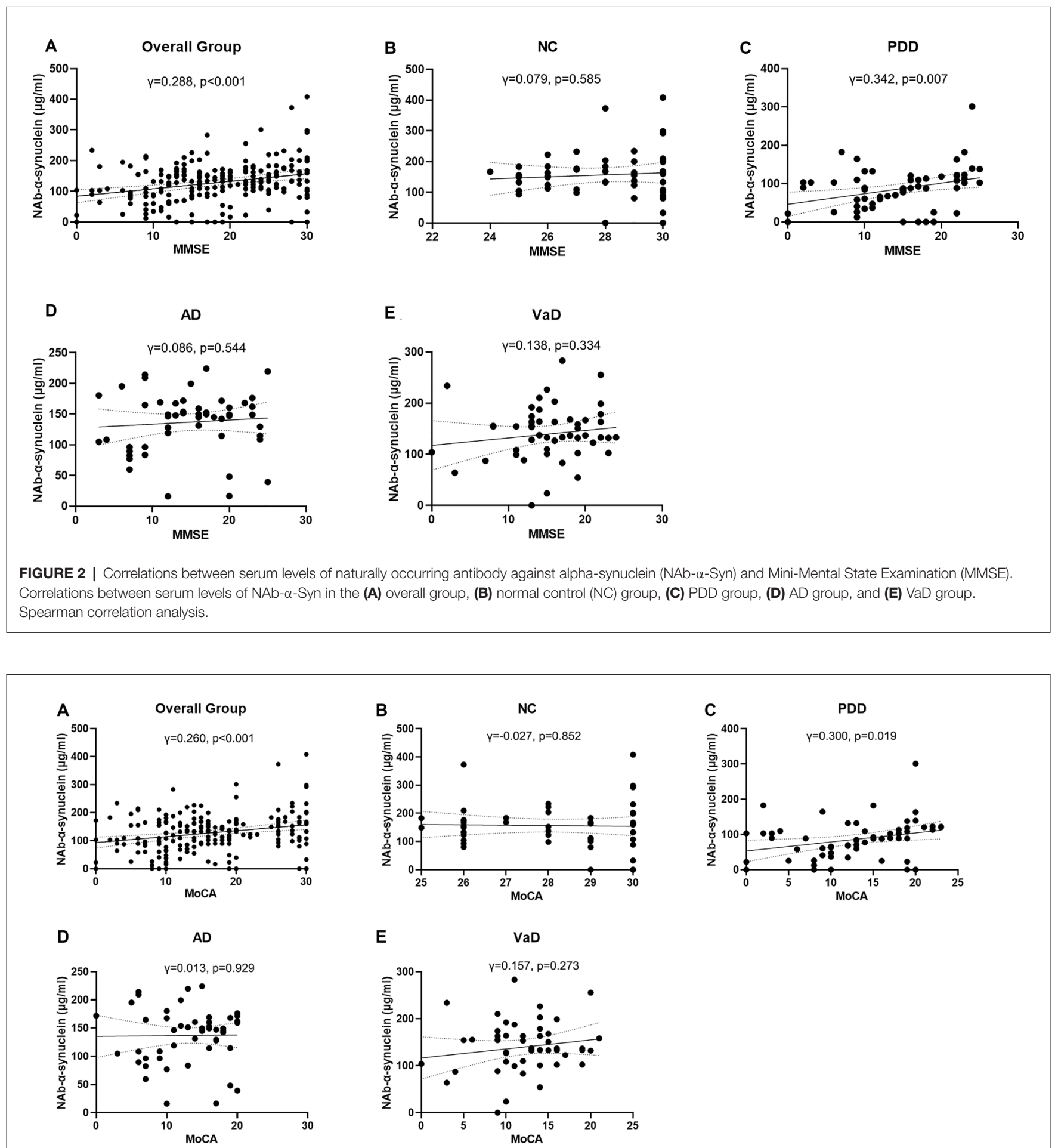

FIGURE 3 | Correlations between serum levels of naturally occurring antibody against alpha-synuclein (NAb- $\alpha$-Syn) and Montreal Cognitive Assessment (MoCA). Correlations between serum levels of NAb- $\alpha$-Syn in the (A) overall group, (B) normal control (NC) group, (C) PDD group, (D) AD group, and (E) VaD group. Spearman correlation analysis.

in the PDD group (Figures 4C,D). However, serum NAb- $\alpha$-Syn levels were not correlated with the duration of dementia in the
$\mathrm{AD}(\gamma=0.051, p=0.718$; Figure 4E) or $\mathrm{VaD}$ group $(\gamma=-0.167$, $p=0.242$; Figure $4 F)$. 

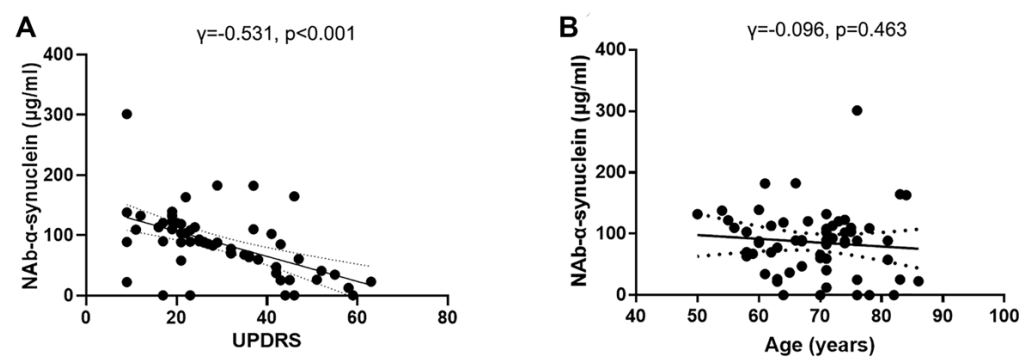

D

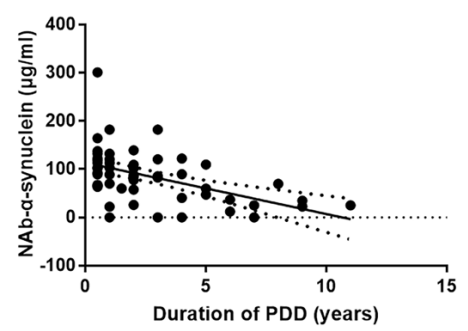

E

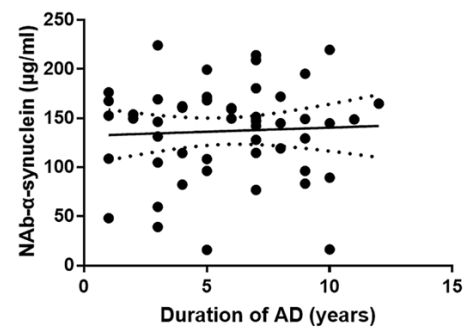

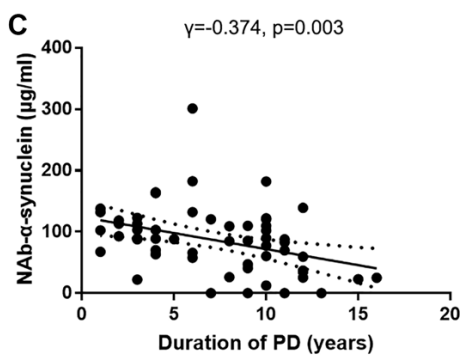

$\mathbf{F}$

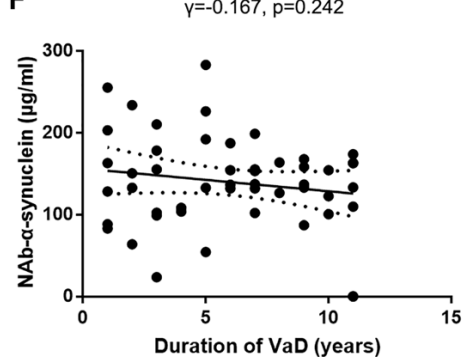

FIGURE 4 | Correlations of serum levels of naturally occurring antibody against alpha-synuclein (NAb- $\alpha$-Syn) with disease severity and duration. (A) Correlation between serum levels of NAb- $\alpha$-Syn and Unified Parkinson Disease Rating Scale (UPDRS) of PDD. (B) Correlation between serum levels of NAb- $\alpha$-Syn and age of PDD. (C) Correlation between serum levels of NAb- $\alpha$-Syn and duration of Parkinson's disease (PD). (D) Correlation between serum levels of NAb- $\alpha$-Syn and duration of PDD. (E) Correlation between serum levels of NAb- $\alpha$-Syn and duration of AD. (F) Correlation between serum levels of NAb- $\alpha$-Syn and duration of VaD. Spearman correlation analysis.

A

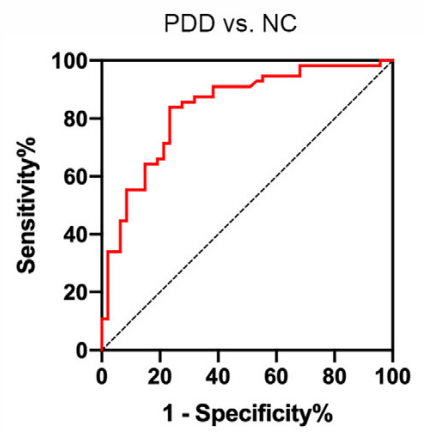

B

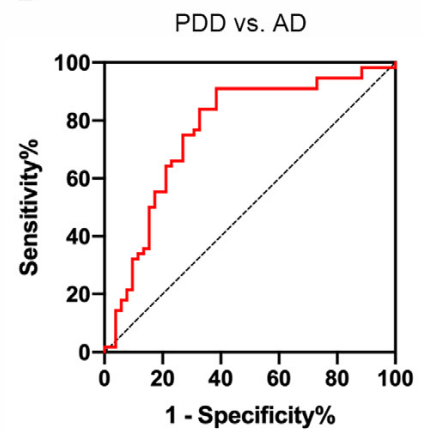

C

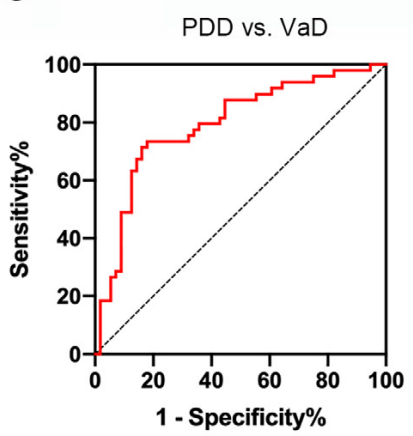

FIGURE 5 | Receiver operating characteristic (ROC) curves of serum naturally occurring antibody against alpha-synuclein (NAb- $\alpha$-Syn) in differentiating PDD from normal control (NC) and other types of dementia. (A) The ROC curve of serum NAb- $\alpha$-Syn in differentiating PDD from NC. (B) The ROC curve of serum NAb- $\alpha$-Syn in differentiating PDD from AD. (C) The ROC curve of serum NAb- $\alpha$-Syn in differentiating PDD from VaD.

\section{The Capacity of Serum Naturally Occurring Antibody Against Alpha-Synuclein in Differentiating Different Types of Dementia}

Using the ROC curve analysis, we found that the area under the ROC curve (AUC) of serum NAb- $\alpha$-Syn in PDD vs. NC was $0.837(p<0.001,95 \%$ confidence interval $=0.758-0.915)$. When applying the optimal cut-off value of $123.0 \mathrm{pg} \mathrm{ml}^{-1}$ calculated using the Youden index, the overall sensitivity and specificity of serum NAb- $\alpha$-Syn for distinguishing PDD from NC was $83.9 \%$ and $76.6 \%$, respectively (Figure 5A). The AUC of serum
NAb- $\alpha$-Syn in PDD vs. AD was $0.712(p<0.001,95 \%$ confidence interval $=0.672-0.859)$. When applying the optimal cut-off value of $140.7 \mathrm{pg} \mathrm{ml} \mathrm{m}^{-1}$, the overall sensitivity and specificity of serum NAb- $\alpha-S y n$ for distinguishing PDD from $\mathrm{AD}$ were $91.1 \%$ and $61.5 \%$, respectively (Figure 5B). The AUC of serum NAb- $\alpha-S y n$ in PDD vs. VaD was 0.794 $(p<0.001,95 \%$ confidence interval $=0.706-0.882)$. When applying the optimal cut-off value of $140.7 \mathrm{pg} \mathrm{ml}^{-1}$, the overall sensitivity and specificity of serum NAb- $\alpha$-Syn for distinguishing PDD from AD were $73.5 \%$ and $82.1 \%$, respectively (Figure 5C). 


\section{DISCUSSION}

In the present study, we found differential serum levels of NAb$\alpha$-Syn among $\mathrm{PDD}, \mathrm{AD}$, and $\mathrm{VaD}$ patients. Serum levels of $\mathrm{NAb}-\alpha-S y n$ were correlated with the cognitive function and disease severity of PDD. Moreover, serum levels of NAb- $\alpha$ Syn is a potential biomarker for differentiating PDD from $\mathrm{AD}$ and $\mathrm{VaD}$.

$\alpha$-Syn exists in its soluble form and can efflux from the brain into the periphery, which may stimulate the production of NAb- $\alpha$-Syn. The levels of NAb- $\alpha$-Syn in PD were controversial in previous studies (Besong-Agbo et al., 2013; Folke et al., 2019). Currently, few studies have investigated the circulating levels of NAb- $\alpha$-Syn in PDD. We found in this study that circulating NAb- $\alpha$-Syn was decreased in PDD patients. However, $\mathrm{AD}$ and $\mathrm{VaD}$ patients had comparable circulating NAb- $\alpha-S y n$ levels. These findings imply that the pattern of circulating NAb$\alpha$-Syn may have disease specificity among neurodegenerative diseases. The mechanism underlying the decrease of NAb- $\alpha$ Syn is not clear yet. We found that serum NAb- $\alpha$-Syn levels were correlated with the duration of PD and PDD, but not with the age of PDD patients, indicating that the alteration of NAb- $\alpha$-Syn levels are not consequent to aging, but to the $\alpha$ Syn pathogenesis. It is possible that NAb- $\alpha$-Syn is consumed by increased circulating $\alpha$-Syn.

The decrease of NAb- $\alpha$-Syn in PDD may have some clinical and pathological relevance. We found that circulating levels of NAb- $\alpha$-Syn were positively correlated with MMSE and MoCA scores in the overall group and PDD subgroup. Furthermore, circulating levels of NAb- $\alpha$-Syn were also negatively correlated with UPDRS in the PDD group. These findings suggest an association between NAb- $\alpha$-Syn and the severity of neurodegeneration in PDD. While the etiology of PDD is multifactorial, $\alpha$-Syn is suggested to be a central component to the pathogenesis of the disease (Rocha et al., 2018). NAb- $\alpha$-Syn may aid in the clearance of $\alpha$-Syn, resulting in the alleviation of neuronal injury by $\alpha$-Syn. This hypothesis is supported by previous studies, which suggests that NAb- $\alpha$-Syn rescues memory and motor functions and attenuates $\alpha$-Syn pathologies in animal models of PD (Huang et al., 2019). In this regard, NAb- $\alpha$-Syn might be a physiological protective factor against $\alpha$-Syn pathologies. The decrease of NAb- $\alpha$-Syn in PDD might contribute to $\alpha$-Syn-induced memory loss and motor dysfunctions.

\section{REFERENCES}

Altomare, D., De Wilde, A., Ossenkoppele, R., Pelkmans, W., Bouwman, F., Groot, C., et al. (2019). Applying the ATN scheme in a memory clinic population: the ABIDE project. Neurology 93, e1635-e1646. doi: 10.1212/WNL. 0000000000008361

Besong-Agbo, D., Wolf, E., Jessen, F., Oechsner, M., Hametner, E., Poewe, W., et al. (2013). Naturally occurring $\alpha$-Synuclein autoantibody levels are lower in patients with Parkinson disease. Neurology 80, 169-175.doi: 10.1212/WNL. 0b013e31827b90d1

El-Agnaf, O. M., Salem, S. A., Paleologou, K. E., Curran, M. D., Gibson, M. J., Court, J. A., et al. (2006). Detection of oligomeric forms of $\alpha$-Synuclein protein
Currently, the differential diagnosis of dementia is largely based on the symptom spectrum of patients. Recent progress in molecular imaging (Janelidze et al., 2020; Kantarci et al., 2020; Kozlova et al., 2020) and cerebrospinal fluid (CSF) biomarkers (Altomare et al., 2019; Llibre-Guerra et al., 2019) improved the diagnostic accuracy of dementia. However, due to the high cost and invasiveness of these diagnostic strategies, bloodbased biomarkers are in urgent need for the diagnosis and differential diagnosis of dementia. Our ROC curve analysis found that circulating NAb- $\alpha$-Syn has relatively high accuracy to differentiate PDD from $\mathrm{AD}$ and $\mathrm{VaD}$. As there is some overlap of clinical manifestations among different types of dementia, NAb$\alpha$-Syn might be a potential differential diagnostic biomarker of PDD.

This study is limited by its cross-sectional nature and small sample size. Besides, this study is a pure correlative study that investigated the association between NAb- $\alpha$-Syn and PDD. However, we found a disease-specific change of circulating NAb$\alpha$-Syn in PDD. This study also proposed a possible role of NAb$\alpha$-Syn in the pathogenesis of PDD from a clinical aspect.

\section{DATA AVAILABILITY STATEMENT}

The raw data supporting the conclusions of this article will be made available by the authors, without undue reservation.

\section{ETHICS STATEMENT}

The studies involving human participants were reviewed and approved by Sichuan Provincial Hospital. The patients/participants provided their written informed consent to participate in this study.

\section{AUTHOR CONTRIBUTIONS}

J-HW and MH designed the study and drafted the manuscript. JW and BZ conducted the experiments, collected and analyzed the data, and drafted the manuscript. SY and $\mathrm{MH}$ were responsible for clinical assessment of the subjects.

\section{FUNDING}

This study was supported by major scientific research project of Sichuan Science and Technology Agency (No. 2019ZYZF0063).

in human plasma as a potential biomarker for Parkinson's disease. FASEB J. 20, 419-425. doi: 10.1096/fj.03-1449com

Emre, M., Aarsland, D., Brown, R., Burn, D. J., Duyckaerts, C., Mizuno, Y., et al. (2007). Clinical diagnostic criteria for dementia associated with Parkinson's disease. Mov. Disord. 22, 1689-1707; quiz 1837. doi: 10.1002/mds.21507

Erkinjuntti, T., Inzitari, D., Pantoni, L., Wallin, A., Scheltens, P., Rockwood, K., et al. (2000). Research criteria for subcortical vascular dementia in clinical trials. J. Neural Transm. Suppl. 59, 23-30. doi: 10.1007/978-3-7091-6781-6_4

Folke, J., Rydbirk, R., Lokkegaard, A., Salvesen, L., Hejl, A. M., Starhof, C., et al. (2019). Distinct autoimmune anti- $\alpha$-Synuclein antibody patterns in multiple system atrophy and Parkinson's disease. Front. Immunol. 10:2253. doi: 10.3389/fimmu.2019.02253 
Hanagasi, H. A., Tufekcioglu, Z., and Emre, M. (2017). Dementia in Parkinson's disease. J. Neurol. Sci. 374, 26-31. doi: 10.1016/j.jns.2017.01.012

Huang, Y. R., Xie, X. X., Ji, M., Yu, X. L., Zhu, J., Zhang, L. X., et al. (2019). Naturally occurring autoantibodies against $\alpha$-Synuclein rescues memory and motor deficits and attenuates $\alpha$-Synuclein pathology in mouse model of Parkinson's disease. Neurobiol. Dis. 124, 202-217. doi: 10.1016/j.nbd.2018. 11.024

Janelidze, S., Mattsson, N., Palmqvist, S., Smith, R., Beach, T. G., Serrano, G. E., et al. (2020). Plasma P-tau181 in Alzheimer's disease: relationship to other biomarkers, differential diagnosis, neuropathology and longitudinal progression to Alzheimer's dementia. Nat. Med. 26, 379-386. doi: 10.1038/s41591-020-0755-1

Kantarci, K., Lowe, V. J., Chen, Q., Przybelski, S. A., Lesnick, T. G., Schwarz, C. G., et al. (2020). $\beta$-Amyloid PET and neuropathology in dementia with Lewy bodies. Neurology 94, e282-e291. doi: 10.1212/WNL.0000000000008818

Kozlova, I., Parra, M. A., Titova, N., Gantman, M., and Sala, S. D. (2020). Alzheimer's disease and parkinson dementia distinguished by cognitive marker. Arch. Clin. Neuropsychol. doi: 10.1093/arclin/acz082 [Epub ahead of print].

Llibre-Guerra, J. J., Li, Y., Schindler, S. E., Gordon, B. A., Fagan, A. M., Morris, J. C., et al. (2019). Association of longitudinal changes in cerebrospinal fluid total tau and phosphorylated tau 181 and brain atrophy with disease progression in patients with Alzheimer disease. JAMA Netw. Open 2:e1917126. doi: 10.1001/jamanetworkopen.2019.17126

McKhann, G. M., Knopman, D. S., Chertkow, H., Hyman, B. T., Jack, C. R. Jr., Kawas, C. H., et al. (2011). The diagnosis of dementia due to Alzheimer's disease: recommendations from the National Institute on Aging-Alzheimer's
Association workgroups on diagnostic guidelines for Alzheimer's disease. Alzheimers Dement. 7, 263-269. doi: 10.1016/j.jalz.2011.03.005

Papachroni, K. K., Ninkina, N., Papapanagiotou, A., Hadjigeorgiou, G. M., Xiromerisiou, G., Papadimitriou, A., et al. (2007). Autoantibodies to $\alpha$ Synuclein in inherited Parkinson's disease. J. Neurochem. 101, 749-756. doi: 10.1111/j.1471-4159.2006.04365.x

Rocha, E. M., De Miranda, B., and Sanders, L. H. (2018). $\alpha$-Synuclein: pathology, mitochondrial dysfunction and neuroinflammation in Parkinson's disease. Neurobiol. Dis. 109, 249-257. doi: 10.1016/j.nbd.2017.04.004

Skovronsky, D. M., Lee, V. M., and Trojanowski, J. Q. (2006). Neurodegenerative diseases: new concepts of pathogenesis and their therapeutic implications. Annu. Rev. Pathol. 1, 151-170. doi: 10.1146/annurev.pathol.1.110304.100113

Tofaris, G. K., and Spillantini, M. G. (2007). Physiological and pathological properties of $\alpha$-Synuclein. Cell. Mol. Life Sci. 64, 2194-2201. doi: 10.1007/s00018-007-7217-5

Conflict of Interest: The authors declare that the research was conducted in the absence of any commercial or financial relationships that could be construed as a potential conflict of interest.

Copyright (c) 2020 Wang, Zheng, Yang, Hu and Wang. This is an open-access article distributed under the terms of the Creative Commons Attribution License (CC BY). The use, distribution or reproduction in other forums is permitted, provided the original author(s) and the copyright owner(s) are credited and that the original publication in this journal is cited, in accordance with accepted academic practice. No use, distribution or reproduction is permitted which does not comply with these terms. 